\title{
Unilateral hemispheric lesions disrupt parallel processing within the contralateral intact hemisphere: an auditory fMRI study
}

\author{
Michela Adriani, ${ }^{\mathrm{a}}$ Anne Bellmann, ${ }^{\mathrm{a}}$ Reto Meuli, ${ }^{\mathrm{b}}$ Eleonora Fornari, ${ }^{\mathrm{b}}$ Rolf Frischknecht, ${ }^{\mathrm{c}}$ \\ Claire Bindschaedler, ${ }^{\mathrm{a}}$ François Rivier, ${ }^{\mathrm{a}}$ Jean-Philippe Thiran, ${ }^{\mathrm{d}}$ \\ Philippe Maeder, ${ }^{\mathrm{b}} \uparrow$ and Stephanie Clarke ${ }^{\mathrm{a}, *} \uparrow$ \\ ${ }^{a}$ Division de Neuropsychologie, CHUV, 1011 Lausanne, Switzerland \\ ${ }^{\mathrm{b}}$ Service de Radiodiagnostic et de Radiologie Interventionnelle, CHUV, 1011 Lausanne, Switzerland \\ ${ }^{\mathrm{c}}$ Service de Rhumatologie et Médecine Réhabilitative, CHUV, 1011 Lausanne, Switzerland \\ ${ }^{\mathrm{d}}$ Institut de Traitement des Signaux, École Polytechnique Fédérale de Lausanne, Lausanne, Switzerland
}

\begin{abstract}
Evidence from activation studies suggests that sound recognition and localization are processed in two distinct cortical networks that are each present in both hemispheres. Sound recognition and/or localization may, however, be disrupted by purely unilateral damage, suggesting that processing within one hemisphere may not be sufficient or may be disturbed by the contralateral lesion. Sound recognition and localization were investigated psychophysically and using fMRI in patients with unilateral right hemisphere lesions. Two patients had a combined deficit in sound recognition and sound localization, two a selective deficit in sound localization, one a selective deficit in sound recognition, and two normal performance in both tasks. The overall level of activation in the intact left hemisphere of the patients was smaller than in normal control subjects, irrespective of whether the patient's performance in the psychophysical tasks was impaired. Despite this overall decrease in activation strength, patients with normal performance still exhibited activation patterns similar to those of the control subjects in the recognition and localization tasks, indicating that the specialized brain networks subserving sound recognition and sound localization in normal subjects were also activated in the patients with normal performance, albeit to an altogether lesser degree. In patients with deficient performance, on the other hand, the activation patterns during the sound recognition and localization tasks were severely reduced, comprising fewer and partly atypical activation foci compared to the normal subjects. This indicates that impaired psychophysical performance correlates with a breakdown of parallel processing within specialized networks in the contralesional hemisphere.
\end{abstract}

(C) 2003 Elsevier Inc. All rights reserved.

Keywords: Auditory cortex; Sound recognition; Sound localization; Diaschisis

\section{Introduction}

Recent studies in human and nonhuman primates demonstrated that auditory information relevant to sound recognition and sound localization is processed in anatomically distinct networks. In macaque auditory cortex three sets of areas called core, belt, and parabelt have been identified (for review see, e.g., Kaas et al., 1999; Rauschecker, 1998). Lateral belt areas-called, from front to back, anterolateral (AL), mediolateral (ML), and caudolateral (CL)—-tend to

\footnotetext{
* Corresponding author. Fax: +41-21-314-13-19.

E-mail address: Stephanie.Clarke@chuv.hospvd.ch (S. Clarke).

$\dagger$ These authors contributed equally to this article.
}

respond to complex sounds corresponding to monkey calls and/or to spatial locations; while area ML is sensitive to both types of information, AL appears more specialized for monkey call-like stimuli and CL for locations (Recanzone, 2000; Tian et al., 2001). AL has been proposed to be part of the auditory stream that subserves sound recognition (the "What" stream) and CL part of the stream that subserves sound localization (the "Where" stream; Rauschecker and Tian, 2000).

A similar functional specialization of nonprimary auditory areas may exist in human. Anatomical (Rivier and Clarke, 1997; Wallace et al., 2002; Chiry et al., 2003) and activation studies (Wessinger et al., 2001) suggest a hierarchical organization of human auditory areas and in partic- 
ular a subdivision into core and belt areas. The posterior part of the planum temporale was found to be involved in spatial processing (Weeks et al., 1999; Warren et al., 2002), the lateral part in sound pattern analysis (Griffiths and Warren, 2002), and the more anterior part of the superior temporal gyrus (on the left side) in speech processing (Binder et al., 2000). Distinct processing streams for sound recognition and sound localization have been identified beyond the early stage auditory areas in the parietal and temporal cortices (Griffiths et al., 1998; Bushara et al., 1999; Weeks et al., 1999; Alain et al., 2001; Maeder et al., 2001). In particular, sound attributes conveying information about sound source identity are selectively processed within a network which involves the temporal convexity bilaterally as well as a part of the left inferior frontal gyrus, while spatial sound attributes are processed within a bilateral network comprising the posterior parietal and prefrontal cortices (Maeder et al., 2001).

Based on their functional studies, some authors suggested a right-hemisphere dominance for auditory spatial processing (Griffiths and Green, 1999; Weeks et al, 1999; Griffiths et al., 2000), whereas others found no evidence for functional lateralization (Bushara et al., 1999; Woldorff et al., 1999). Unilateral hemispheric lesions were shown to impair the ability to localize sound sources. Several studies reported mislocalization within the hemispace contralateral to the brain lesion, after right or left hemispheric lesions (Wortis and Pfeffer, 1948; Sanchez-Longo and Forster, 1958; Klingon and Bontecou, 1966; Poirier et al., 1994; Zatorre et al., 1995). Other studies described deficits within the whole field following unilateral lesions (Haeske-Dewick et al., 1996; Zatorre and Penhune, 2001) and some advocated either right (Ruff et al., 1981; Bisiach et al., 1984; Tanaka et al., 1999) or left (Pinek et al., 1989) hemispheric specialization for auditory localization.

Activation studies demonstrated that categorization of environmental sounds involves more specifically left prefrontal, temporal, parietal, and cingulate regions (Engelien et al., 1995) while another showed bilateral involvement of regions on the temporal and prefrontal convexities (Maeder et al., 2001). The ability to recognize environmental sounds was found to be deficient following unilateral right (Spreen et al., 1965; Assal and Aubert, 1979; Fujii et al., 1990; Clarke et al., 1996) or left (Pasquier et al., 1991; Clarke et al., 2000) hemisphere lesions.

Thus, networks subserving sound recognition and sound localization in human have been demonstrated in both hemispheres, with only marginal hemispheric differences (Maeder et al., 2001). However, sound recognition and/or localization may be disrupted by purely unilateral damage (see above), suggesting that processing within one hemisphere may either not be sufficient to sustain normal function or be disrupted by the contralateral lesion. We have addressed these hypotheses by investigating performance in sound localization and sound recognition and the corre- sponding activation patterns in patients with unilateral right hemisphere lesions.

\section{Materials and methods}

Seven patients with a first unilateral right hemisphere lesion who participated in the neuropsychological rehabilitation program of the Division of Neuropsychology were included in the study (Table 1). These patients met the following criteria: (i) no prior neurological or psychiatric illness, (ii) normal hearing thresholds in tonal audiometry, (iii) right-handedness, and (iv) absence of major behavioral deficits. Informed consent of the patients and control subjects was obtained according to the Declaration of Helsinki. The study was approved by the Ethics Committee for Clinical Research, Medical Faculty, University of Lausanne.

Sound recognition and sound localization were investigated with tests, which were digitally constructed on a Power Macintosh 8100 equipped with an audio-media card and the software Protools Powermix and Sound Designer II. During the testing session the patients sat in a quiet room in front of the examiner. The stimuli were played through earphones linked to the computer and set at the volume judged comfortable by the patients.

\section{Semantic recognition of environmental sounds}

Recognition of sounds was tested by presenting the patient with 50 samples, each of which lasted $7 \mathrm{~s}$. Each sound was presented with a multiple-choice display of five drawings: the target, an object acoustically and semantically related to the sound, a semantically related object, an acoustically related object, and an object neither acoustically nor semantically related to the sound. The patient had to point out the correct drawing. A detailed description of the test and normative data on 60 normal subjects were published previously (Clarke et al., 1996).

\section{Auditory localization}

Localization was tested by simulating different azimuthal positions of a sound source by varying interaural time difference (ITD). The stimulus was a 2 -s broadband bumblebee sound, shaped with 100-ms rising and falling times. One central and four lateral positions were simulated, two in each hemispace, using 0.3- and 1-ms time differences with either the left or the right channel leading. The task contained 60 items, 12 in each position, presented in pseudo-random order. As a measure of overall performance, the relative positions attributed to two consecutive stimuli were compared; a response was counted as correct when a stimulus was correctly placed to the left or the right of the previous stimulus in correspondence with the difference in ITD or within $\pm 10^{\circ}$ of the previous location for identical ITD. The average relative score obtained by the control 
Table 1

Performance in sound recognition and sound localization in seven patients with focal right hemisphere lesions

\begin{tabular}{|c|c|c|c|c|c|c|c|c|}
\hline \multirow[t]{2}{*}{ Case } & \multirow[t]{2}{*}{ Age } & \multirow[t]{2}{*}{ Sex } & \multirow[t]{2}{*}{ Handedness } & \multirow[t]{2}{*}{ Etiology } & \multirow[t]{2}{*}{ Site of lesion } & \multirow[t]{2}{*}{ Time from lesion } & \multicolumn{2}{|c|}{ Auditory tasks } \\
\hline & & & & & & & Loc & $\operatorname{Rec}$ \\
\hline \multicolumn{9}{|c|}{ Deficient performance } \\
\hline 1 & 46 & $\mathrm{~F}$ & Right & Ischemia & F-P, Ins, BG & $\begin{array}{l}\text { Tests: } 2 \text { months } \\
\text { fMRI: } 3 \text { months }\end{array}$ & -10.122 & -3.625 \\
\hline 2 & 28 & $\mathrm{~F}$ & Right & Ischemia & F-P-T, Ins, BG, CC & $\begin{array}{l}\text { Tests: } 2 \text { months } \\
\text { fMRI: } 2.5 \text { months }\end{array}$ & -5.660 & -2.401 \\
\hline 3 & 47 & M & Right & Ischemia & F-P-T, Ins, BG, CC & $\begin{array}{l}\text { Tests: } 13 \text { months } \\
\text { fMRI: } 13 \text { months }\end{array}$ & -3.430 & -1.972 \\
\hline 4 & 46 & M & Right & Ischemia & F-P-T, Ins, CC & $\begin{array}{l}\text { Tests: } 3 \text { months } \\
\text { fMRI: } 4 \text { months }\end{array}$ & $-2.000^{\mathrm{a}}$ & 0.040 \\
\hline 5 & 50 & $\mathrm{~F}$ & Right & Ischemia & F-P-T, Ins, CC & $\begin{array}{l}\text { Tests: } 1 \text { month } \\
\text { fMRI: } 2 \text { months }\end{array}$ & -1.750 & -2.401 \\
\hline \multicolumn{9}{|c|}{ Normal performance } \\
\hline 6 & 40 & $\mathrm{~F}$ & Right & Ischemia & F-P-T, Ins, BG, CC & $\begin{array}{l}\text { Tests: } 1 \text { month } \\
\text { fMRI: } 2.5 \text { months }\end{array}$ & -0.083 & 0.047 \\
\hline 7 & 23 & M & Left & $\begin{array}{l}\text { Epidural hematoma } \\
\text { (evacuated) }\end{array}$ & $\begin{array}{l}\text { No visible lesion at } \\
\text { testing }\end{array}$ & $\begin{array}{l}\text { Tests: } 3 \text { weeks } \\
\text { fMRI: } 1 \text { month }\end{array}$ & -0.083 & -0.360 \\
\hline
\end{tabular}

Lesions were analyzed on structural MRI scans. Performances are expressed in $Z$ scores relative to control population $(n=60)$; deficient results are in bold. BG, basal ganglia; CC, corpus callosum or intrahemispheric part of the callosal pathway; F, frontal; Ins, insula; P, parietal; T, temporal.

${ }^{a}$ Deficient performance additionally characterized by a high variability in attributing one position to a given ITD and inability to discriminate two positions within one hemispace, on both the right and the left side; neither of these errors was observed in the normal subjects.

population was 57.15 correct responses of 59 possible. Detailed descriptions of the test and normative data on 60 subjects were published previously (Clarke et al., 2000; Bellmann et al., 2001; Bellmann, Thiran, and Clarke, 2003).

\section{Lesion analysis}

Lesions were analyzed on structural MRI scans acquired in the same session as functional MRI (Table 1, Fig. 1). The normalized coordinate system of Talairach and Tournoux (1988) was adopted for the comparison of sites of lesions with activation foci observed in normal subjects in a previous study. Lesions were delineated on structural scans and analyzed using OrthoViewer, an image visualization and processing software, developed in one of our laboratories. The software can display orthogonal views of a 3D image, after manual detection of Talairach transformation landmarks.

\section{fMRI paradigm}

Brain activation associated with sound recognition or sound localization was investigated as in a previous study with normal subjects (Maeder et al., 2001). Three conditions were used: (i) comparison of sound location simulated with interaural time differences, (ii) identification of environmental sounds, and (iii) rest. Conditions (i) and (ii) required a motor response. Activation patterns were analyzed using SPM99 for individual subjects.

Stimuli for conditions (i) and (ii) lasted $5 \mathrm{~s}$ and they preceded the image acquisition; they consisted of a background and a sound target. In the recognition task, the background consisted of an everyday auditory scene (marketplace, street, beach, shop, railway station); targets were animal cries. In the localization task the background consisted of 25 white noise bursts, each lasting $50 \mathrm{~ms}$, with interaural time differences varying between 0 and $681 \mu \mathrm{s}$; targets were two 500-ms tokens of white noise tracts (lowpass filtered of $1000 \mathrm{~Hz}$ ), the first presented $1.5 \mathrm{~s}$ and the second $3 \mathrm{~s}$ after the onset of the background. The task was to discriminate whether the second target was presented at the same or a different location in comparison to the first one. Sound generation, sound transmission, and response monitoring were done as in the previously published study on normal subjects (Maeder et al., 2001).

BOLD fMRI acquisitions were performed with a head coil on a 1.5-T Siemens Magnetom Vision system equipped for echoplanar imaging. Sixteen slices, $5 \mathrm{~mm}$ thick with 1-mm gap, covering the whole brain in the bicommissural plane were acquired with an EPI gradient echo T2*weighted sequence (FA 90, TE 66; pixel size $1.8 \times 1.8 \mathrm{~mm}$, acquisition time $3.95 \mathrm{~s}$ ). A TR of $15 \mathrm{~s}$ and precise adjustment of the stimulus offset and acquisition onset guaranteed that fMRI data were acquired while the BOLD effect was elicited by the stimuli (sparse sampling technique; Belin et al., 1999; Hall et al., 1999 same as in Maeder et al., 2001). A sagittal conventional T1-weighted 3D gradient-echo sequence (MPRAGE), 128 slices $1.25 \mathrm{~mm}$ thick, was acquired as the structural basis for lesion analysis and Talairach transformation. 


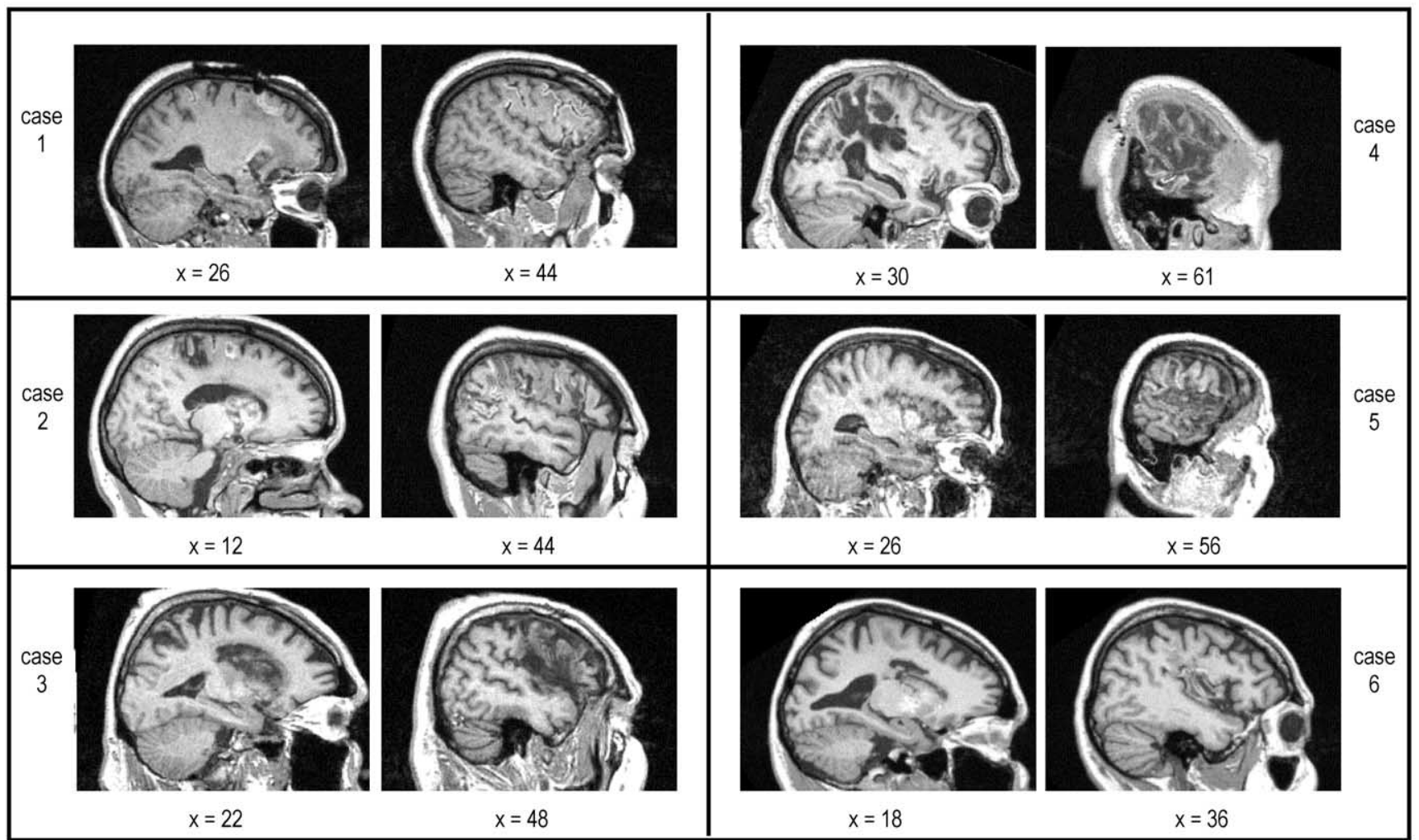

Fig. 1. Lesions of patients 1-6, shown in T1-weighted MRI scans. The Talairach and Tournoux (1988) coordinates of the parasagittal planes are indicated.

Preprocessing and data analysis were done on a Silicon Graphic Indigo 2 workstation with SPM99 (Wellcome Department of Cognitive Neurology, London, UK). First, the images of each subject were realigned in order to correct for head movement during acquisition. All images were then normalized matching each image to a Montreal Neurological Institute template and resliced to a $2 \times 2 \times 2$-mm voxel size using the bilinear interpolation method. A smoothing procedure was applied in order to increase the signal-tonoise ratio using an isotropic Gaussian kernel of 6-mm FWHM. Statistical analysis was performed on each subject according to the general linear model as implemented in SPM99; baseline drift across the imaging time series was attenuated with a high-pass filter and changes in global activity were removed by proportional scaling. Contrasts of interest (recognition vs rest, localization vs rest, recognition vs localization, localization vs recognition) were estimated using a weighted square-wave function, and the respective hypotheses were tested with a $t$ statistic generating a statistical parametrical map $\operatorname{SPM}\{t\}$ of corresponding $T$ values for each voxel. Regions significantly activated were considered to be those who survived a threshold of $T=3.19$ ( $p<$ 0.001 uncorrected with $d f=81$ ) and contained a cluster of at least 60 contiguous activated voxels. This is the same statistical analysis as was used in our previous study on normal subjects (Maeder et al., 2001; Figs. 2A and 2B).

\section{Results}

Performance in sound localization and sound recognition

Four patients (cases 1-4) were deficient in sound localization (see Table 1); two were also deficient in sound recognition (cases 1 and 2). One patient was deficient in sound recognition but normal in sound localization (case 5), and two patients were normal in both sound localization and sound recognition (cases 6 and 7).

\section{Sites and extents of lesions}

Lesions of patients 1-6 involved to different extents the frontoparietal and temporal cortices, the basal ganglia, the insula, and/or the callosal pathway (Table 1). They encroached (Fig. 1), to different extents, upon regions known to be involved in sound recognition and sound localization, which have been described in a previous study in normal subjects (Maeder et al., 2001) and are shown in Figs. 2A and $2 \mathrm{~B}$.

Damage to auditory structures shared by the auditory What and Where streams (the auditory thalamus, the acoustic radiation, the primary auditory area and the surrounding auditory regions that were equally activated by the sound recognition and sound localization tasks in normal subjects, 
A

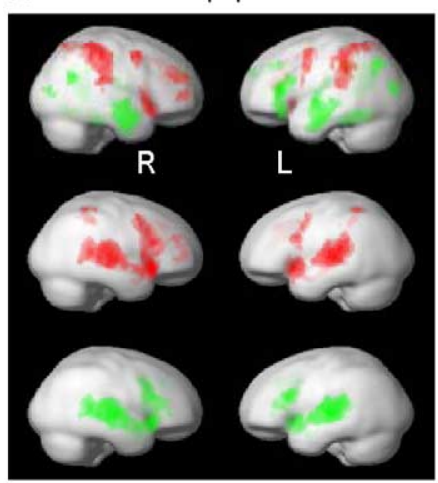

D

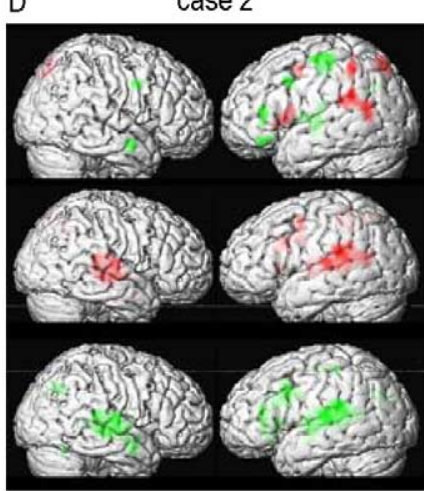

G

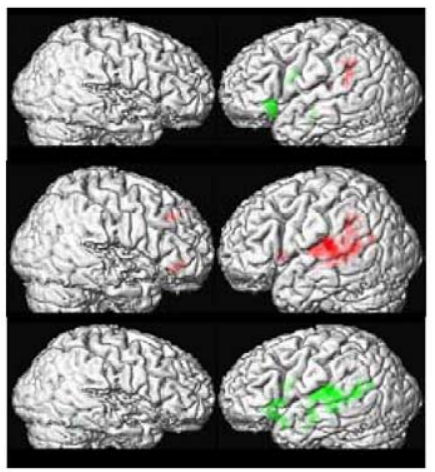

B

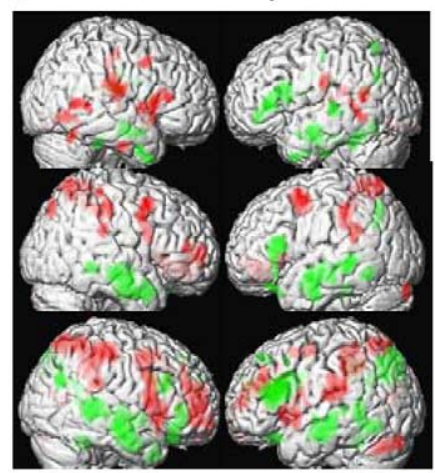

E

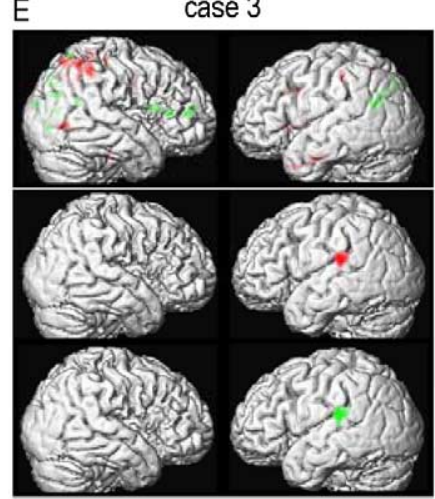

$\mathrm{H}$

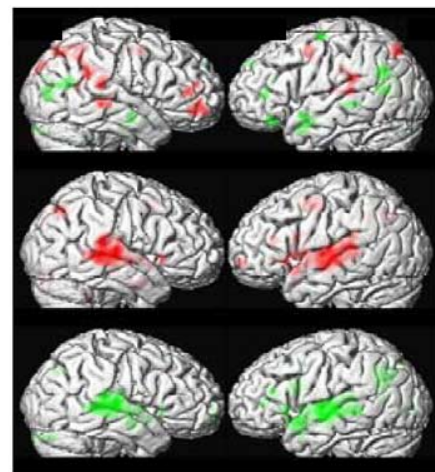

C

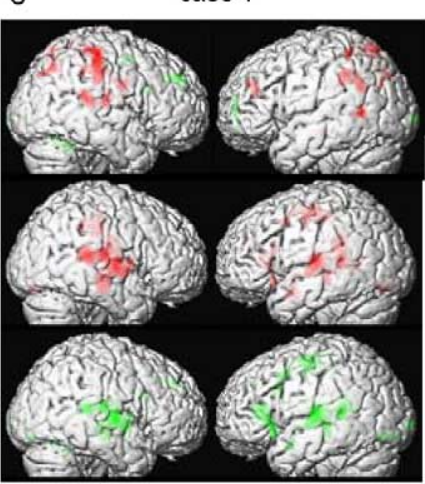

F

case 4

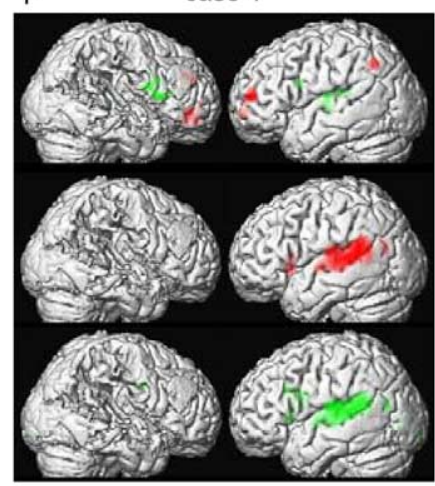

I

case 7

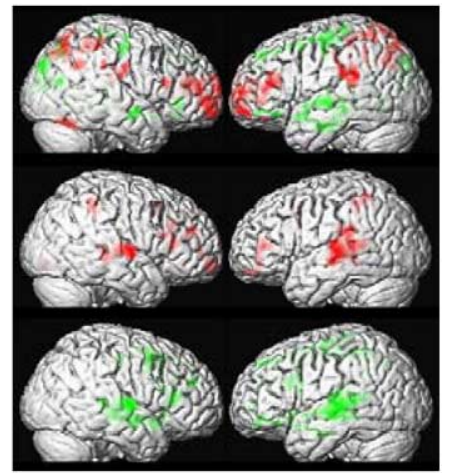

Fig. 2. Cortical networks involved in sound localization and sound recognition in normal subjects (A and B; from our previous study, Maeder et al., 2001, reproduced by permission of the publisher) and in patients 1-7 (C-I). (A) Group study of 18 normal subjects showing networks selectively involved in sound localization (red) or sound recognition (top: red for localization $>$ recognition, green for recognition $>$ localization), networks globally involved in localization (middle: red for localization $>$ rest), and networks globally involved in recognition (bottom; green for recognition $>$ rest). (B) Interindividual variations (three normal subjects) in networks selectively involved in sound localization and sound recognition (red for localization $>$ recognition, green for recognition > localization). (C-I) Individual patient data showing networks selectively involved in sound localization (red) or sound recognition (top: red for localization $>$ recognition, green for recognition $>$ localization), networks globally involved in localization (middle: red for localization $>$ rest), and networks globally involved in recognition (bottom: green for recognition $>$ rest). Same level of significance was used for B-I and a comparable level in the population study shown in A $(T=3.19, p=0.001, K=60)$. In B-I the three-dimensional reconstruction of the brain surface of the corresponding subject is shown. $\mathrm{R}$ and $\mathrm{L}$ in the first row of A indicate right and left hemisphere, respectively; the same convention is used for all other images.

the auditory callosal pathway, and in particular the paraventricular region), as well as to the auditory What (the temporal convexity) and Where streams (the inferior parietal lobule), has been evaluated on structural MRI scans.

Cases 1 and 3 had a partial lesion of the shared auditory structures and a large lesion of the Where stream; case 3 but not case 1 had a lesion of the auditory callosal pathway.
Case 2 had a partial lesion of the shared auditory structures and the What stream as well as a large lesion of the Where stream and of the auditory callosal pathway. Case 4 had a very large lesion which destroyed most parts of the shared auditory structures, the What and Where streams, and the auditory callosal pathway. Case 5 had a partial lesion of the What stream and a large lesion of the shared auditory 
structures, the Where stream, and the auditory callosal pathway. Case 6 had a partial lesion of the Where stream and a disconnection of the auditory callosal pathway. Case $7 \mathrm{did}$ not present any detectable lesion on MRI at the time of testing, but sustained a massive epidural frontoparietal hematoma on the right side 3 weeks earlier, which necessitated surgical evacuation.

A good association between damage to specialized networks and performance was observed in the one patient who was tested more than a year after the lesion occurred (case 3 ). His lesion was centered on the frontoparietal cortex and involved thus mainly the Where stream and spared the temporal convexity where key parts of the What stream are located; he had the corresponding deficit in sound localization, while sound recognition was relatively preserved. This was not always the case in four patients who were tested in the postacute phase. Two patients with a combined lesion of the frontotemporoparietal cortex, the insula, and the callosal pathway, involving the shared auditory structures and both the What and the Where streams, had an isolated deficit in sound localization (case 4) or sound recognition (case 5). Another patient (case 1), with a lesion centered on the frontoparietal cortex, the insula, and the basal ganglia, involving the shared auditory structures and the Where stream, had a combined deficit of sound localization and sound recognition. One patient (case 2), with a lesion centered on the frontotemporoparietal cortex and involving mainly the Where stream, presented a combined deficit of sound localization and sound recognition. Thus, only one of four cases (case 2) had the auditory deficit pattern that we would expect from observation in chronic cases. A similar lack of specificity between the deficit and the damage to a specialized network was reported in the acute stage (Adriani et al., 2003).

All patients but case 1 had an auditory callosal disconnection, due to right paraventricular lesions.

\section{Activation patterns elicited by sound localization and sound recognition}

The contrast between the auditory tasks and the rest condition yielded no activation in the right damaged hemisphere of two patients (cases 3 and 4), while spared parts of the right hemisphere were activated in the other five patients (1, 2, 5-7; Figs. 2C-2I, middle and bottom). In all patients, the amount of right hemisphere activation by auditory tasks vs rest was below, or at the lower limit of, that observed in normal subjects (Fig. 3), as would be expected, since the lesions were in the right hemisphere. Surprisingly, however, the left, structurally intact hemisphere also tended to be less activated by the auditory tasks in patients than in normal subjects. Localization vs rest activated the left hemisphere below (cases 1, 3-5, 7) or at the lower limit of (cases 2 and 6) what has been observed in normal subjects. Recognition vs rest activated the left hemisphere also below (cases 3 and
5), at the lower limit of (cases 1, 4, and 7), or in the lower range of (cases 2 and 6) normal activation levels.

Thus, a unilateral lesion decreases activation in the contralesional, intact hemisphere, in patients with both deficient and normal performance in psychophysical auditory tests. The question arises as to how far this decrease in overall activation level is accompanied by lesion-related changes in the activation patterns characteristic of sound recognition and sound localization in normal subjects.

Specialized networks for sound recognition and sound localization failed to be activated normally in the right and left hemispheres of all five patients with a deficit in sound recognition and/or sound localization (cases 1-5; Fig. 2). In our previous study all 18 normal subjects had a cluster activated by recognition vs localization on the temporal convexity bilaterally and a cluster activated by localization vs recognition in the inferior parietal lobule bilaterally (Figs. 2A and 2B). This was not the case in patients with deficient performance in sound localization and/or sound recognition (Figs. 2C-2G). Case 3 did not have any of these four clusters. Three other cases failed to activate the temporal recognition clusters bilaterally and presented only the parietal activation on the left side (cases 4 and 5) or bilaterally (case 1). Case 2 had a small temporal recognition cluster on the right side and a small parietal localization cluster on the left side.

Changes in the activation patterns for the localization vs recognition and recognition vs localization contrast within the intact, left hemisphere did not, however, correlate with patterns of deficits. Four of the five patients had a small localization cluster in the inferior parietal lobule, irrespective of whether they had a combined deficit in sound localization and sound recognition (cases 1 and 2), a selective deficit in sound localization (case 4), or a selective deficit in sound recognition (case 5). The patient who had no selective activation associated with the auditory tasks on the left side had a selective deficit in sound localization (case 3).

Despite the overall decrease in right and left activation levels, the specialized networks in the two patients with normal performance in sound localization and sound recognition (cases 6 and 7) were very similar to those in normal subjects. Both patients activated temporal recognition and parietal localization clusters bilaterally, as did normal subjects.

\section{Discussion}

Our results show that unilateral hemispheric lesions influence the contralateral processing of auditory information in two different ways: (i) by an overall decrease of activation in the damaged as well as the intact hemisphere, irrespective of the presence of performance deficits, and (ii) changes in task-specific activation patterns indicating a breakdown of parallel processing within specialized networks, which correlates with deficient performance. 

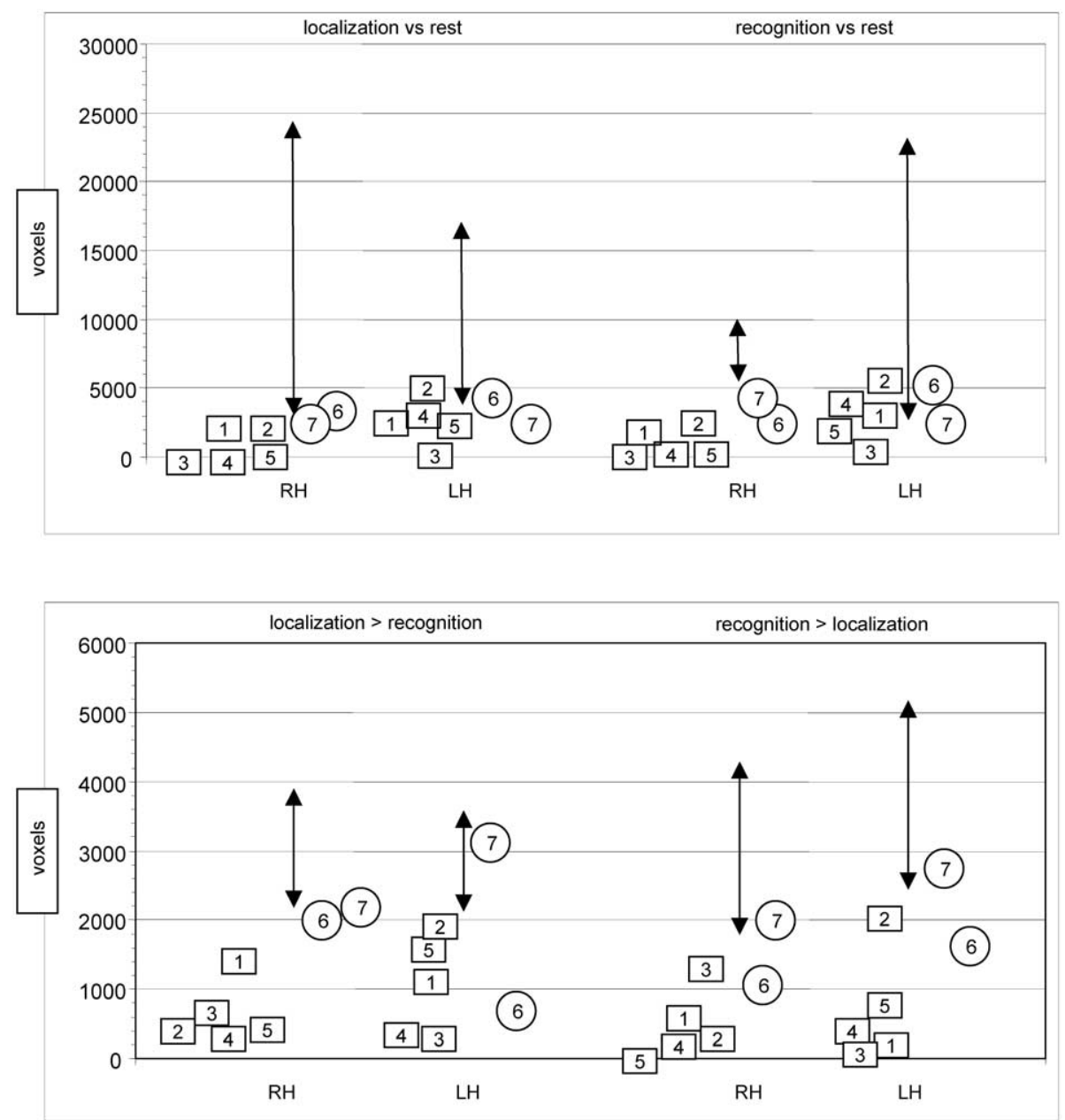

Fig. 3. Amount of activation (number of activated voxels) in the right (RH) and left hemispheres (LH) in four different conditions (localization vs rest, recognition vs rest, localization $>$ recognition, and recognition $>$ localization) for patients with a deficient performance (squares) who presented a combined deficit in sound recognition and sound localization (cases 1 and 2), a selective deficit in sound localization (cases 3 and 4), or a selective deficit in sound recognition (case 5) and for patients with normal performance (circles) in sound recognition and sound localization (cases 6 and 7). Arrows indicate the range found in 18 normal subjects (from Maeder et al., 2001).

The decrease in overall activation level in the intact, contralateral hemisphere following a purely unilateral damage has been described in previous studies assessing glucose metabolism (Fiorelli et al., 1991) or cerebral blood flow (Lenzi et al., 1982; Lagreze et al., 1987; Dobkin et al., 1989). We demonstrated here a decrease in activation by a specific auditory task in the intact hemisphere. This decrease occurred both in patients with a deficient performance and in patients with a normal performance and thus does not correlate with the degree of putative recovery. Previous studies investigating recovery of functions that rely predominantly on unilateral networks (aphasia, neglect, hemiplegia) have stressed the correlation between activation foci in the intact hemisphere and improved function (e.g., Pizzamiglio et al., 1998; Weiller, 1998). The decreased activation that we have observed is more likely to be found in the context of bilaterally represented (nonlateralized) networks. A unilateral lesion may have disturbed the anatomically remote parts of the network (as has been demon- strated very elegantly for unilateral networks; Price and Friston, 1999; Barcelo et al., 2000; Price et al., 2001). In our cases, the presence or absence of deficits is not linked to the overall decrease in activation, but to the degree of functional integration that the undamaged parts of the network can still sustain.

In our paradigm, the functional correlate of deficient vs normal performance is the breakdown of parallel processing within specialized networks of the otherwise intact hemisphere. Patients with deficient performance in sound localization and/or sound recognition had a low degree of selective activation by the localization or by the recognition task. Furthermore, key parts of the localization and recognition networks, which are present in all normal subjects $(n=18$; Maeder et al., 2001) were not at all, or were only partially, activated. The activation patterns in the intact left hemisphere did not correlate consistently with preserved sound localization or sound recognition. A part of the key parietal localization cluster was activated in patients with a com- 
bined deficit in sound localization and sound recognition (cases 1 and 2), a selective deficit in sound localization (case 4), or a selective deficit in sound recognition (case 5). A part of the key temporal recognition cluster failed to be activated in patients with a selective deficit in sound recognition (case 5) or a combined deficit in sound recognition and sound localization (cases 1 and 2), but also in two patients with preserved sound recognition (but deficient sound localization; cases 3 and 4). Thus, fully preserved parallel processing within the intact hemisphere is necessary for preserved sound recognition and sound localization.

The mechanisms of loss of parallel processing within the auditory What and Where networks, as observed in this study, are currently unknown. The auditory information is relayed to the intact hemisphere via the acoustic radiation and the primary auditory area. From there it is distributed to the nonprimary areas in a parallel and hierarchical fashion, feeding into the What and Where networks (e.g., Chiry et al., 2003). The processing within auditory areas is most likely modulated by a complex system of interhemispheric connections, including homo- and heterotopic afferents ( $\mathrm{Di}$ Virgilio and Clarke, 1997; Di Virgilio et al., 1999). Partial or complete loss of the interhemispheric input, due to the contralateral lesion, may change the routing of auditory information and result in the processing of auditory recognition and localization in the same cortical areas. Synaptic reshaping of the remaining interhemispheric inputs, including subcortical ones (e.g., via the superior colliculus; Tardif et al., 2002) as well as of the intrinsic connections (Tardif and Clarke, 2000) may contribute to the restoration of parallel processing in some cases.

\section{Conclusion}

Sound recognition and sound localization are sustained by bilaterally distributed specialized networks. Unilateral, here right, hemispheric lesions disturbed processing in the contralateral, intact hemisphere by (i) decreasing activation by sound recognition and sound localization tasks, independent of the performance level of the patient, and (ii) breaking down parallel processing within the specialized networks, which was then associated with deficient performance.

\section{Acknowledgments}

This study was supported by Swiss National Science Foundation Grants 3138-51173.97 and 3238-064604.01 and by a Lausanne Medical Faculty RATP grant to S.C.

\section{References}

Adriani, M., Maeder, P., Meuli, R., Bellmann Thiran, A., Frischknecht, R., Villemeure, J.-G., Mayer, J., Annoni, J.-M., Bogousslavsky, J., Fornari,
E., Thiran, J.-P., Clarke, S., 2003. Sound recognition and localisation in man: specialised cortical networks and effects of acute circumscribed lesions. Exp. Brain Res. (in press).

Alain, C., Arnott, S.R., Hevenor, S., Graham, S., Grady, C.L., 2001. "What" and "Where" in the human auditory system. Proc. Natl. Acad. Sci. USA 98, 12301-12306.

Assal, G., Aubert, C., 1979. Recognition of onomatopoeias and animal cries during focalized lesions of the cerebral cortex. Rev. Neurol. (Paris) 135, 65-73.

Barceló, F., Suwazono, S., Knight, R.T., 2000. Prefrontal modulation of visual processing in humans. Nat. Neurosci. 3, 399-403.

Belin, P., Zatorre, R.J., Hoge, R., Evans, A.C., Pike, B., 1999. Eventrelated fMRI of the auditory cortex. NeuroImage 10, 417-429.

Bellmann, A., Meuli, R., Clarke, S., 2001. Two types of auditory neglect. Brain 124, 676-687.

Binder, J.R., Frost, J.A., Hammeke, T.A., Bellgowan, P.S.F., Springer, J.A., Kaufman, J.N., Possing, E.T., 2000. Human temporal lobe activation by speech and nonspeech sounds. Cereb. Cortex 10, 512-528.

Bisiach, E., Cornacchia, L., Sterzi, R., Vallar, G., 1984. Disorders of perceived auditory lateralization after lesions of the right hemisphere. Brain 107, 37-52.

Bushara, K.O., Weeks, R.A., Ishii, K., Catalan, M.J., Tian, B., Rauschecker, J.P., Hallett, M., 1999. Modality-specific frontal and parietal areas for auditory and visual spatial localization in humans. Nat. Neurosci. 2, 759-766.

Chiry, O., Tardif, E., Magistretti, P.J., Clarke, S., 2003. Patterns of calcium-binding proteins support parallel and hierarchical organization of human auditory areas. Eur. J. Neurosci. 17, 1-14.

Clarke, S., Bellmann, A., de Ribaupierre, F., Assal, G., 1996. Non-verbal auditory recognition in normal subjects and brain-damaged patients: evidence for parallel processing. Neuropsychologia 34, 587-603.

Clarke, S., Bellmann, A., Meuli, R.A., Assal, G., Steck, A.J., 2000. Auditory agnosia and auditory spatial deficits following left hemispheric lesions: evidence for distinct processing pathways. Neuropsychologia 38, 797-807.

Di Virgilio, G., Clarke, S., 1997. Direct interhemispheric visual input to human speech areas. Hum. Brain Mapp. 5, 347-354.

Di Virgilio, G., Clarke, S., Pizzolato, G., Schaffner, T., 1999. Cortical regions contributing to the anterior commissure in man. Exp. Brain Res. 124, 1-7.

Dobkin, J.A., Levine, R.L., Lagreze, H.L., Dulli, D.A., Nickles, R.J., Rowe, B.R., 1989. Evidence for transhemispheric diaschisis in unilateral stroke. Arch. Neurol. 46, 1333-1336.

Engelien, A., Silbersweig, D., Stern, E., Huber, W., Doring, W., Frith, C., Frackowiak, R.S., 1995. The functional anatomy of recovery from auditory agnosia. A PET study of sound categorization in a neurological patient and normal controls. Brain 118, 1395-1409.

Fiorelli, M., Blin, J., Backhine, S., Laplane, D., Baron, J.C., 1991. PET studies of cortical diaschisis in patients with motor hemi-neglect. J. Neurol. Sci. 104, 135-142.

Fujii, T., Fukatsu, R., Watabe, S., Ohnuma, A., Teramura, K., Kimura, I., Saso, S., Kogure, K., 1990. Auditory sound agnosia without aphasia following a right temporal lobe lesion. Cortex 26, 263-268.

Griffiths, T.D., Green, G.R., 1999. Cortical activation during perception of a rotating wide-field acoustic stimulus. NeuroImage 10, 84-90.

Griffiths, T.D., Green, G.G.R., Rees, A., Rees, G., 2000. Human brain areas involved in the analysis of auditory movement. Hum. Brain Mapp. 9, 72-80.

Griffiths, T.D., Rees, G., Rees, A., Green, G.G., Witton, C., Rowe, D., Buchel, C., Turner, R., Frackowiak, R.S., 1998. Right parietal cortex is involved in the perception of sound movement in humans. Nat. Neurosci. $1,74-79$.

Griffiths, T.D., Warren, J.D., 2002. The planum temporale as a computational hub. Trends Neurosci. 25, 348-353.

Haeske-Dewick, H., Canavan, A.G., Homberg, V., 1996. Sound localization in egocentric space following hemispheric lesions. Neuropsychologia 34, 937-942. 
Hall, D.A., Haggard, M.P., Akeroyd, M.A., Palmer, A.R., Summerfield, A.Q., Elliott, M.R., Gurney, E.M., Bowtell, R.W., 1999. "Sparse" temporal sampling in auditory fMRI. Hum. Brain Mapp. 7, 213-223.

Kaas, J.H., Hackett, T.A., Tramo, M.J., 1999. Auditory processing in primate cerebral cortex. Curr. Opin. Neurobiol. 9, 164-170.

Klingon, G.H., Bontecou, D.C., 1966. Localization in auditory space. Neurology 16, 879-886.

Lagrese, H.L., Levine, R.L., Pedula, K.L., Nickles, R.J., Sunderland, J.S., Rowe, B.R., 1987. Contralateral flow reduction in unilateral stroke: evidence for transhemispheric diaschisis. Stroke 18, 882-886.

Lenzi, G.L., Frackowiak, R.S., Jones, T., 1982. Cerebral oxygen metabolism and blood flow in human cerebral ischemic infarction. J. Cereb. Blood Flow Metab. 2, 321-335.

Maeder, P., Meuli, R., Adriani, M., Bellmann, A., Fornari, E., Thiran, J.-P., Pittet, A., Clarke, S., 2001. Distinct pathways involved in sound recognition and localization: a human fMRI study. NeuroImage 14, 802 816.

Pasquier, F., Leys, D., Steiling, M., Guieu, J.D., Petit, H., Cambier, J., 1991. Agnosie auditive unilatérale droite consécutive à une hémorragie lenticulaire gauche. Rev. Neurol. (Paris) 147, 129-137.

Pinek, B., Duhamel, J.-R., Cavé, C., Brouchon, M., 1989. Audio-spatial deficits in humans: differential effects associated with left versus right hemisphere parietal damage. Cortex 25, 175-186.

Pizzamiglio, L., Perani, D., Cappa, S.F., Vallar, G., Paolucci, S., Grassi, F., Paulesu, E., Fazio, F., 1998. Recovery of neglect after right hemispheric damage. Arch. Neurol. 55, 561-568.

Poirier, P., Lassonde, M., Villemure, J.-G., Geoffroy, G., Lepore, F., 1994. Sound localization in hemispherectomized patients. Neuropsychologia $32,541-553$

Price, C.J., Friston, K.J., 1999. Scanning patients with tasks they can perform. Hum. Brain Mapp. 8, 102-108.

Price, C.J., Warburton, E.A., Moore, C.J., Frackowiak, R.S.J., Friston, K.J., 2001. Dynamic diaschisis: anatomically remote and context-sensitive human brain lesions. J. Cognit. Neurosci. 13, 419-429.

Rauschecker, J.P., 1998. Parallel processing in the auditory cortex of primates. Audiol. Neurootol. 3, 86-103.

Rauschecker, J.P., Tian, B., 2000. Mechanisms and streams for processing of "What" and "Where" in auditory cortex. Proc. Natl. Acad. Sci. USA 97, 11800-11806.

Recanzone, G.H., 2000. Spatial processing in the auditory cortex of the macaque monkey. Proc. Natl. Acad. Sci. 97, 11829-11835.

Rivier, F., Clarke, S., 1997. Cytochrome oxidase, acetylcholinesterase, and NADPH-diaphorase staining in human supratemporal and insular cortex: evidence for multiple auditory areas. NeuroImage 6, 288-304.
Ruff, R.M., Hersh, N.A., Pribram, K.H., 1981. Auditory spatial deficits in the personal and extrapersonal frames of reference due to cortical lesions. Neuropsychologia 19, 435-443.

Sanchez-Longo, L.P., Forster, F.M., 1958. Clinical significance of impairment of sound localization. Neurology 8, 119-125.

Spreen, O., Benton, A.L., Fincham, R.W., 1965. Auditory agnosia without aphasia. Arch. Neurol. 13, 84-92.

Talairach, J., Tournoux, P., 1988. Co-planar stereotaxic atlas of the human brain. Thieme, New York.

Tanaka, H., Hachisuka, K., Ogata, H., 1999. Sound lateralization in patients with left or right cerebral hemispheric lesions: relation with unilateral visuospatial neglect. J. Neurol. Neurosurg. Psychiatry 67, 481-486.

Tardif, E., Clarke, S., 2001. Intrinsic connectivity of human auditory areas: a tracing study with DiI. Eur. J. Neurosci. 13, 1045-1050.

Tardif, E., Clarke, S., 2002. Commissural connections of human superior colliculus. Neuroscience 111, 363-372.

Tian, B., Reser, D., Durham, A., Kustov, A., Rauschecker, J.P., 2001. Functional specialization in rhesus monkey auditory cortex. Science 292, 290-293.

Wallace, M.N., Johnston, P.W., Palmer, A.R., 2002. Histochemical identification of cortical areas in the auditory region of the human brain. Exp. Brain Res. 143, 499-508.

Warren, J.D., Zielinski, B.A., Green, G.G.R., Rauschecker, J.P., Griffiths, T.D., 2002. Perception of sound-source motion by the human brain. Neuron 34, 139-148.

Weeks, R.A., Aziz-Sultan, A., Bushara, K.O., Tian, B., Wessinger, C.M., Dang, N., Rauschecker, J.P., Hallett, M., 1999. A PET study of human auditory spatial processing. Neurosci. Lett. 262, 155-158.

Weiller, C., 1998. Imaging recovery from stroke. Exp. Brain Res. 123, 13-17.

Wessinger, C.M., VanMeter, J., Tian, B., Van Lare, J., Pekar, J., Rauschecker, J.P., 2001. Hierarchical organization of the human auditory cortex revealed by functional magnetic resonance imaging. J. Cognit. Neurosci. 13, 1-7.

Woldorff, M.G., Tempelmann, C., Fell, J., Tegeler, C., Gaschler-Markefski, B., Hinrichs, H., Heinze, H.J., Scheich, H., 1999. Lateralized auditory spatial perception and the contralaterality of cortical processing as studied with functional magnetic resonance imaging and magnetoencephalography. Hum. Brain Mapp. 7, 49-66.

Wortis, S.B., Pfeffer, A.Z., 1948. Unilateral auditory-spatial agnosia. J. Nerv. Ment. Dis. 108, 181-186.

Zatorre, R.J., Penhune, V.B., 2001. Spatial localization after excision of human auditory cortex. J. Neurosci. 21, 6321-6328.

Zatorre, R.J., Ptito, A., Villemure, J.-G., 1995. Preserved auditory spatial localization following cerebral hemispherectomy. Brain 118, 879-889. 